\title{
Associational effects in the microbial neighborhood
}

\author{
Anja Worrich ${ }^{1} \cdot$ Niculina Musat $\mathbb{D}^{2} \cdot$ Hauke Harms ${ }^{1,3}$
}

Received: 14 January 2019 / Revised: 8 May 2019 / Accepted: 8 May 2019 / Published online: 21 May 2019

(c) International Society for Microbial Ecology 2019

\begin{abstract}
Even though "perfect" metagenomes or metatranscriptomes are close at hand, the implicit assumption of spatial homogeneity in the "omic" approaches makes it difficult if not impossible to relate those data to ecological processes occurring in natural and man-made ecosystems. In fact, the distribution of microbes in their habitats is far from being uniform and random. Microbial communities show a high degree of spatial organization that stems from environmental gradients and local interactions. These interactions can be very complex and may involve multiple species. Several studies highlighted the importance of indirect interactions for community stability, but the absence of a theoretical framework for microbial ecology restricts the possibilities to strike a balance between the investigation of simple communities with purely pairwise interactions and the attempts to understand interaction patterns in whole communities based on meta-omics studies. Here we suggest adapting the concept of Associational Effects (AE) from plant ecology, to better understand the link between ecological interactions, spatial arrangement, and stability in microbial communities. By bringing together a conceptual framework developed for plants and observations made for microbes, this perspective article fosters synthesis of related disciplines to yield novel insights into the advancing field of spatial microbial ecology. To promote the integration into microbial ecology, we (i) outline the theoretical background of AE, (ii) collect underlying mechanisms by literature synthesis, (iii) propose a three-point roadmap for the investigation of $\mathrm{AE}$ in microbial communities, and (iv) discuss its implications for microbial ecology research.
\end{abstract}

\section{A way forward in spatial microbial ecology}

In a world beyond well-mixed culture flasks, microorganisms live in multispecies communities with distinct patterns of spatial organization [1]. Although these patterns are considered a main force driving the composition and function of microbial communities, field and laboratory studies often neglect the role of spatial complexity. However, microbes communicate and interact with their neighbors via physical, chemical, and biological mechanisms. The spatial structure of microbial communities facilitates

Anja Worrich

anja.worrich@ufz.de

1 Department of Environmental Microbiology, UFZ - Helmholtz Centre for Environmental Research, Permoserstrasse 15, 04318 Leipzig, Germany

2 Department of Isotope Biogeochemistry, UFZ - Helmholtz Centre for Environmental Research, Permoserstrasse 15, 04318 Leipzig, Germany

3 German Centre for Integrative Biodiversity Research (iDiv) HalleJena-Leipzig, Deutscher Platz 5e, 04103 Leipzig, Germany and consolidates these interactions and provides organisms with advantages for growth and adaptation under fluctuating environmental conditions [2].

Although the important role of the spatial context is wellknown in ecology, the methodological difficulties associated with observing microbes in nature at interactionrelevant scales impeded a rapid progress in the field of spatial microbial ecology. The advances in microscopy, the fabrication of tailored microhabitats, and the new era of chemical microscopy and imaging mass spectrometry now enable researchers to observe microbial interactions with high resolution and in complex matrices [3]. Nevertheless, only a few studies exist, which integrated spatial complexity into the investigation of microbial interactions, and all of them focused on direct pairwise interactions in low complexity communities. Indirect interactions involving multiple species were largely neglected in microbial ecology, although several theoretical studies emphasized their importance for species coexistence and community stability [4]. Here we argue that a theoretical framework is needed to move forward with the investigation of these two different axes of complexity in microbial communities studies. The ecological concept of Associational Effects (AE) describes 
the influence of plant neighborhood composition on the interaction between plants and herbivores. This framework has not been matched to microbial ecology, although it opens new opportunities for moving beyond communities with simple bipartite interactions in well-mixed environments to complex and spatially structured communities intertwined by dense interaction networks.

\section{AE: having "good" or "bad" neighbors}

Consumer-resource interactions were extensively studied in plant ecology. Due to the high economical and societal value of many plant species, their interactions with pests and pathogens received a lot of attention fostering the development of numerous ecological concepts. One of those concepts is the framework of $\mathrm{AE}$, which emerged from the observation that consumer-resource interactions can be modified by neighboring organisms. AE occur in plant-herbivore, predator-prey, and plant-pollinator interactions [5]. The main difference to other basic ecological interactions (e.g., competition, mutualism, amensalism, $[\ldots])$ is that $\mathrm{AE}$ represent a form of indirect interaction, in which the presence of a modulator species (plant neighbor) influences the interaction between a focal (plant) and an effector (herbivore) species. The modulation can occur via interaction chains or via higher-order interactions (HOI). The distinction between these two types of interactions is determined by whether the indirect effect emerges from a change in herbivore density (interaction chain) or a change in the "per capita" competitive effects between herbivore and focal species (HOI) [4]. The peculiarity of the AE concept is that the outcome for the focal species can be either positive or negative, and that it takes into account the identity of species in the direct neighborhood of the focal species at particular spatial scales [6]. This distinguishes the concept from other types of indirect interactions, such as "apparent competition", denoting a purely negative indirect interaction between species through shared natural enemies without explicit consideration of spatial aspects [7]. Two opposite AE phenomena are described: either the association increases (Associational Susceptibility, AS) or decreases (Associational Resistance, AR) the vulnerability of the focal plant species (Fig. 1). Understanding this linkage between indirect interactions and spatial arrangement in plant-herbivore interactions yielded ecologically guided agricultural practices to protect crops from herbivore attack. This is achieved by using adjacent plants within the crop plots or by planting patches of non-crop plants next to the focal crop plot [5].

In microbial ecology, however, there is a much broader interest in the manifold types of interactions occurring between different species in a community. Disentangling these dense interaction networks is almost impossible and reductionist approaches are used to understand community dynamics by decomposing the community into smaller and more manageable subsets [8]. There is increasing consensus that the dynamic behavior of a full community is unpredictable based on observations of interactions between subsets of species. To overcome this issue, holistic approaches often focus on diversity as a cause of success or failure of a community with respect to stability or productivity [9]. However, we have to take diversity relationships as a starting point to unravel what specific ecological mechanisms underpin communities' emergent properties. Matching the conceptual framework of $\mathrm{AE}$ to microbial ecology is an attempt to bridge the holistic-reductionist divide [8] to move beyond communities consisting of simple bipartite interactions to more complex, spatially structured communities with numerous direct and indirect interactions. By generalizing the $\mathrm{AE}$ concept with respect to the number of modulator species and the type of interactions between focal and effector species, we integrate the idea of context-dependent microbial interactions influenced by biotic (modulator species) and abiotic (spatial structure) factors. Thus, it will help to gain a better understanding of the behavior of species embedded in complex microbial communities and the role of spatial organization in shaping their interaction patterns. Accordingly, we here adapt the definition of $\mathrm{AE}$ for microbial ecology as follows: $\mathrm{AE}$ can be defined as the impact of one species (effector) on another (focal) that is mediated by other community members (modulators). The outcome depends on the spatial organization of the community members and the type of interaction between effector and focal species. The impact of this modulation on the focal species can be positive (Associational Benefit, $\mathrm{AB}$ ) or negative (Associational Detriment).

Indeed, studies in plant ecology inherently consider the spatial context by placing plants at certain positions in pots or in the field or by analyzing their natural distribution. Microbial ecological experiments, however, are often carried out in shaken liquid cultures ("well-mixed environment") or apply sampling techniques that destroy the spatial organization of the microbial community. Therefore, studies on $\mathrm{AE}$ in microbial ecology have to consider the explicit spatial configuration of the interacting species (Fig. 1).

\section{Mechanisms underlying $A E$ in microbial communities}

Physical, chemical, and biological mechanisms can evoke $\mathrm{AE}$ in microbial communities (Fig. 2). In a study on a predatory bacterium, high diversity of the prey community reduced predation. The authors hypothesized that predationresistant bacteria form barriers around sensitive bacteria and 


\section{The Framework of Associational Effects}

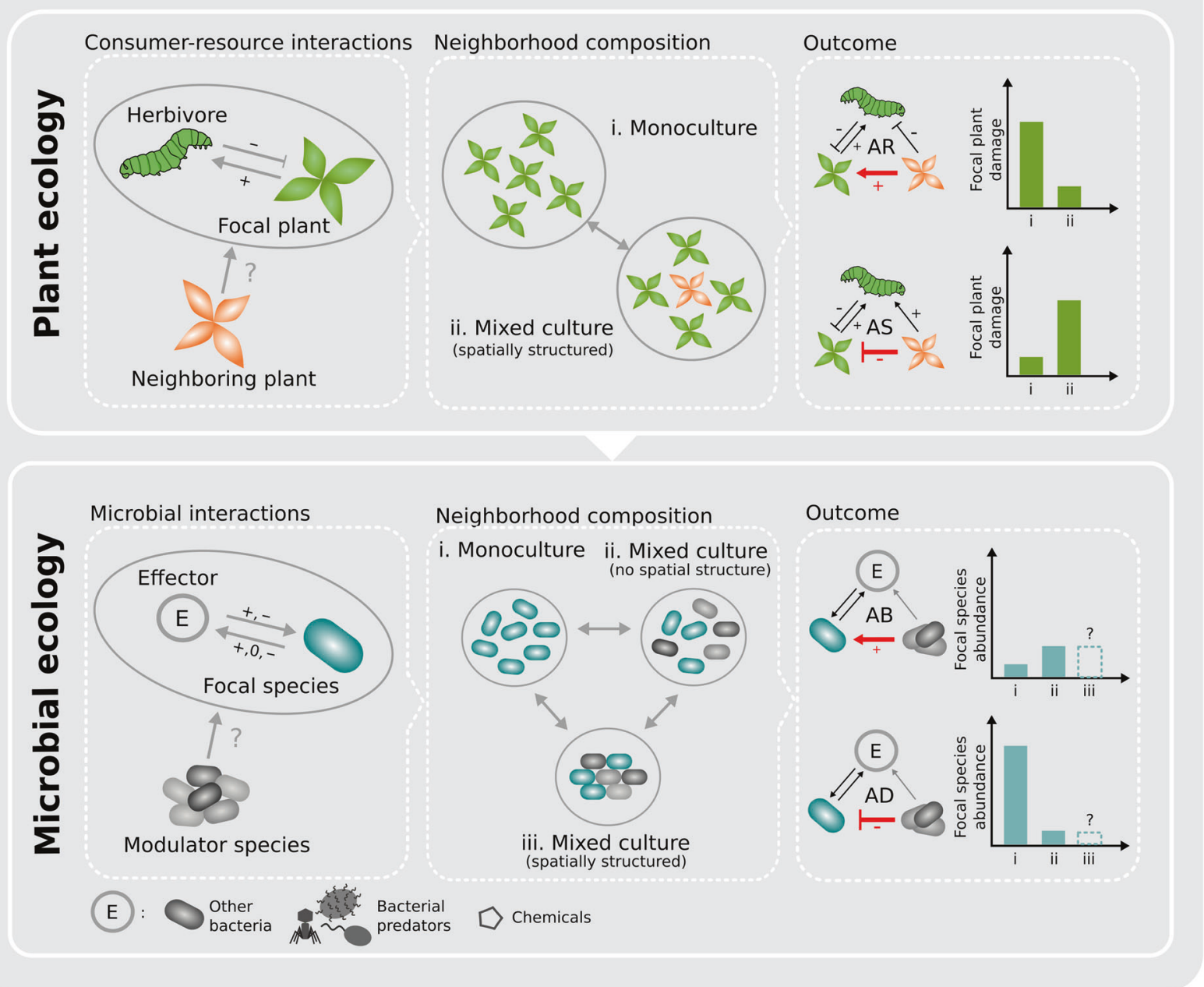

Fig. 1 Transfer of the AE framework from plant to microbial ecology. The framework describes the modulation of the interaction between a plant and a herbivore by a neighboring plant species. The modulation depends on the spatial organization and can either decrease
(Associational Resistance, AR) or increase (Associational Susceptibility, AS) the damage/mortality of the focal species. In microbial ecology, this concept will help to bundle research activities on indirect interactions in spatially structured microbial communities thereby physically shield them from the predator [10]. Biofilm formation alters the physical environment of bacteria and provides collective cell protection against phage or protist attack [11, 12]. Resource competition between community members can induce bacterial cell aggregation due to changes in their surface properties. Bacterial cells living in aggregates are physically protected against predation, as predators cannot reach bacteria in the inner part of the aggregate [13].

In addition to inducing changes in the physical properties, bacteria also shape their chemical environment. The excretion of metabolic byproducts such as lactic acid or antibiotics can affect the growth of other microbes and potentially also of predators. Volatile organic compounds (VOCs) emitted by bacteria serve as weapons in microbial warfare and are effective against various antagonists including fungi, protists, and other bacteria [14]. In unsaturated soils, VOCs act over greater spatial scales than soluble compounds and probably also protect VOC-insensitive bacteria in the surrounding of the producing population.

The production of defensive secondary metabolites is costly and requires an allocation of resources to secondary metabolism [11]. Growth-defense tradeoffs result in lower numbers of individuals. In contrast, the excretion of metabolic byproducts or the release of extracellular enzymes and scavenging molecules can enhance substrate availability in the environment leading to higher cell densities. Sometimes predators concentrate on abundant prey types and this frequency-dependent selection can increase or reduce predation. In a study on phage epidemics in a bacterial population, the immunity strongly depended on the relative frequency of resistant and susceptible individuals, and the 


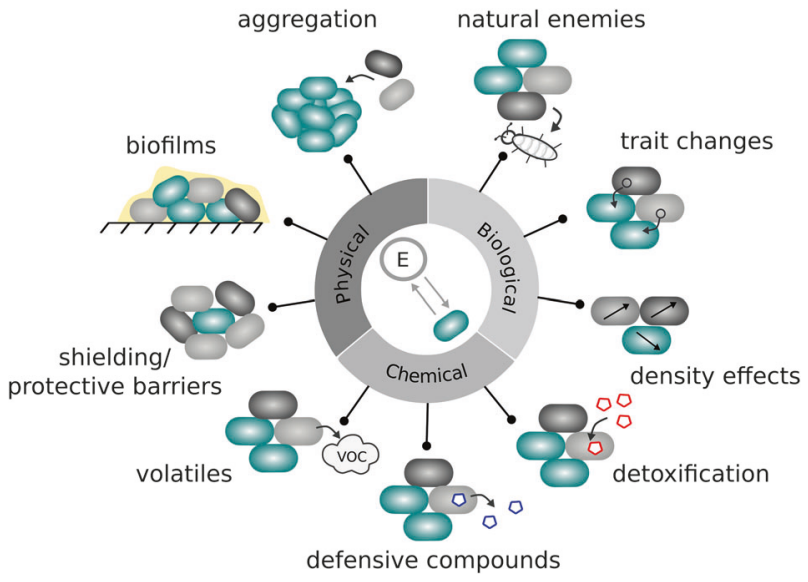

Fig. 2 Physical, chemical, and biological mechanisms causing AE in bacterial communities. Physical mechanisms mainly prevent the accessibility or ingestion of the focal species (F) by forming larger or more complex structures together with the modulating species (M). Chemical mechanisms lead to repellence or attraction of predators via the production and release of secondary metabolites by $\mathrm{M}$, whereas the detoxification of chemicals by $\mathrm{M}$ decreases the susceptibility of $\mathrm{F}$. Biological mechanisms are diverse and include changes in the abundance or traits of $\mathrm{F}$, or the attraction of natural enemies by $\mathrm{M}$

spatial population structure [15]. Although bacteria and phage were able to coexist in a structured habitat, they collapsed in a well-mixed environment due to the low frequency of the resistant individuals [15].

Predator feeding and associated damage of neighboring cells often causes a release of chemical cues that may induce or prime defenses in the focal species. In addition, released compounds may also attract natural enemies of the predator-an indirect defense mechanism well-known for plant VOCs emitted upon plant damage. Although there is no study on the attraction of natural enemies of predators by prey bacteria, bacterial volatiles were already shown to be involved in such complex interaction networks. In a collaborative attempt, two soil bacteria produced VOCs triggering the growth and motility of a Pseudomonas strain, which in turn produced antibacterial and antifungal compounds to the benefit of the VOC producers [16]. Effects of VOCs on bacterial motility, which is a key trait in predator-prey interactions, were observed in many different studies [11] and indicate the occurrence of VOCmediated AE.

The previous section emphasizes that it is rather easy to find examples for $\mathrm{AB}$ in microbial ecology literature. In contrast, fewer studies look at the potential negative effects that modulators can have on cooperating species. Pande et al. [17] showed that non-cooperating bacteria can exploit public goods produced by a cross-feeding consortium leading to a reduced productivity of the latter. Also here, spatial structure had an important impact on the outcome of the modulation. Unlike in liquid cultures, spatial segregation on agar surfaces protected the cross-feeders from being exploited by non-cooperators [17].

\section{Expansion of the concept to abiotic stressors}

AE served as a concept to explain changes in the vulnerability of plants to herbivores and, more recently, the concept has been expanded to herbivore-herbivore associations and their vulnerability to predators, pathogens, parasites, or parasitoids [5]. Here we suggest to adapt the AE concept not only to biotic interactions in microbial ecology but also to the effects of abiotic stressors on bacteria growing embedded in complex communities in spatially structured environments. The rationale is that most of the mechanisms highlighted in Fig. 2 can be responsible for $\mathrm{AE}$ in the presence of both abiotic and biotic stressors. Conceptually, the interaction can be seen as a form of amensalism with the stressor replacing the effector species. The stressor inflicts harm to the focal species without any effect on the stressor itself. The modulator species can affect the extent to which stressors exert negative effects on the focal species by shielding the focal species, by reducing the stress, e.g., via degradation of toxic compounds, or by changing the physiology of the focal species. Depending on the mechanism involved, spatial organization can increase or weaken the effects of the stressor.

This expansion will help to gain a more thorough understanding of the role of compositional and spatial complexity for stability properties of microbial communities. In a recent publication, Frost et al. [18] found that antibiotic-resistant strains provide resistance to sensitive cells when they are nearby. This observation points to the occurrence of $\mathrm{AB}$ in bacterial colonies, in which neighbors shield cells of the focal species and thereby reduce their vulnerability towards abiotic stress. This hypothesis was supported with an individual-based simulation model of microbial biofilms where antibiotic detoxification by resistant cells protected sensitive cells in close vicinity [19]. Such spatial feedbacks were most pronounced in crossfeeding consortia exhibiting a high degree of intermixing of resistant and sensitive cells. In contrast, competitioninduced spatial segregation lifted the benefits of detoxification for the sensitive strain [19].

Another mechanism was observed for Streptococcus mutans populations showing a higher tolerance to various antibacterial agents when present in a mixed biofilm with Veillonella parvula than as a mono-species biofilm. Transcription analysis revealed that $V$. parvula induced changes in gene expression within $S$. mutans and thereby alters its physiology to enhance antibiotic resistance [20]. In addition to spatial shielding and trait changes, $\mathrm{AB}$ can also result from changes in resource availability. Recently, Leisner et al. [21] proposed that antibiotic-resistant cells can take 


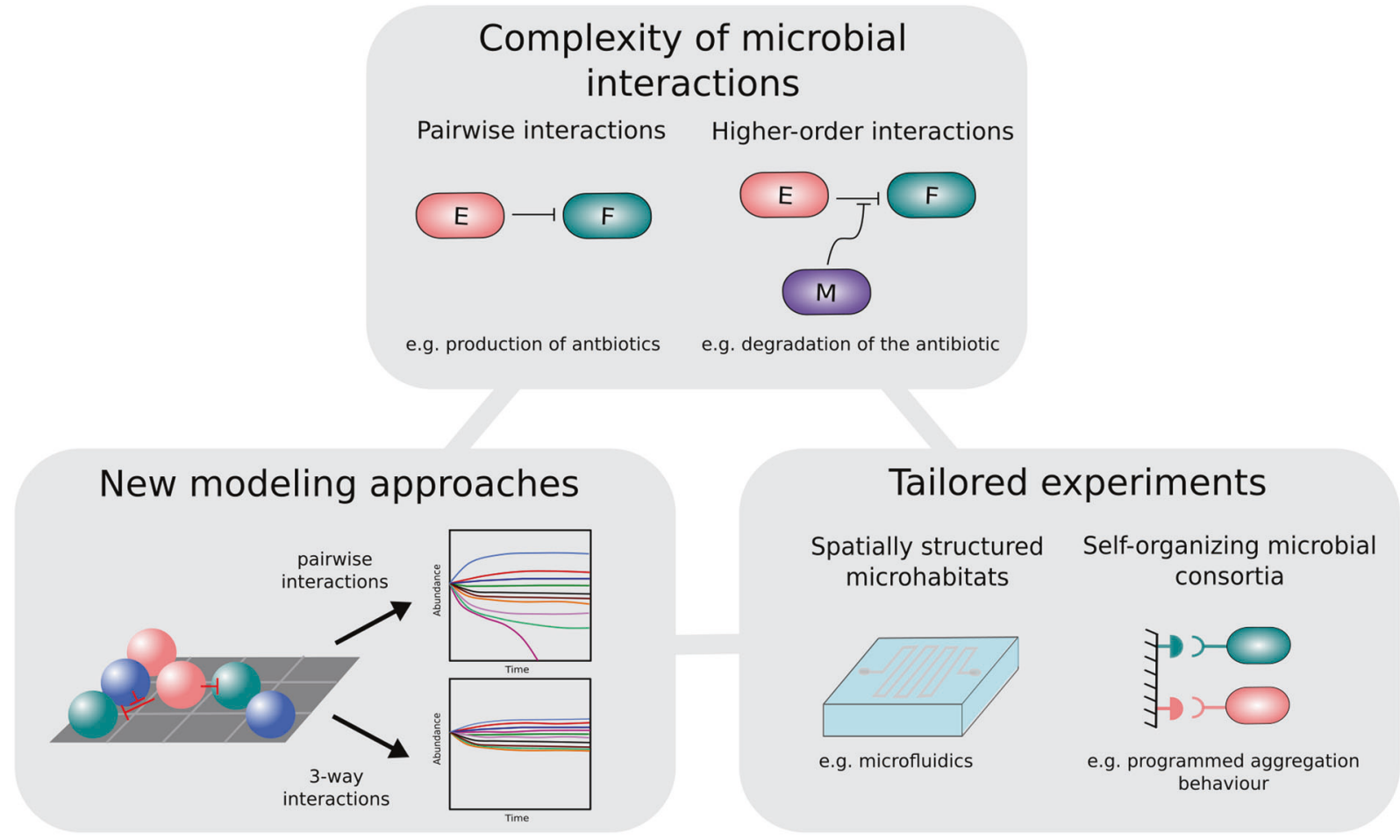

Fig. 3 Conceptual, experimental, and methodological demands to study AE in microbial communities. Although direct pairwise interactions were intensively studied, the importance of indirect interactions in microbial ecology remains elusive. To move beyond communities with simple pairwise interactions, it requires (i) a

advantage of substrates released from lysed sensitive strains to compensate for the fitness costs associated to the maintenance of resistance.

In addition to negative effector chemicals such as antibiotics, the expansion could also comprise chemicals that have a positive effect on the focal species (e.g., signaling molecules). Degradation of quorum-sensing signals by other bacteria can disrupt the communication among populations and is therefore discussed as a prospective therapy option for bacterial diseases [22].

\section{Conceptual, experimental, and methodological developments to study AE in microbial communities}

To study $\mathrm{AE}$ in microbial ecology, at first we have to consider the complexity of microbial communities with respect to both spatial structure and interactions between the community members. There are numerous theoretical studies on the interlinkage between spatial structure and microbial interactions. However, experimental data validating specific hypotheses generated by modeling is scarce. Indeed, just a limited number of studies focused on the interplay of spatial organization of microbial communities rethinking of existing theories and concepts, (ii) tailored experimental systems to study microbial interactions in spatially structured assemblies and habitats, and (iii) new modeling approaches considering higher-order interactions in spatially explicit modeling environments

and the interactions occurring in the community [23-25]. These studies follow the reductionist approach focusing on two species and their pairwise interactions. A conceptual rethinking in terms of multiple species being involved in interactions, however, must be accompanied by experimental and methodological efforts to be able to detect and describe AE in microbial communities (Fig. 3).

\section{Experimental requirements to study $\mathrm{AE}$}

Although individual-based modeling can predict phenomena at the population level as an emergent property of single-cell behaviors, experimental studies need to adapt the analysis to an interaction-relevant scale, which may span from single cells to populations growing in spatially distinct microbial communities (e.g., microcolonies). Single-cell imaging now allows measuring the scale over which individual cells exert an influence on neighboring cells and to define an interaction distance [26] as a direct microscopic measure. In the last years, many different experimental approaches and concepts were developed, which allow for the cultivation and observation of bacteria displaying distinct spatial structures. Fabricated microhabitats and microfluidics were recently highlighted for their potential to overcome the challenges in studying complex systems. 
They bear the potential to manipulate microbial interactions by tailored designs and spatiotemporal control of microbe entry [27]. Soft-lithographic techniques create microstructures by printing, molding, and embossing. Thereby, reproducible patterns can be created varying in the explicit neighborhood arrangement. Recently, the potential of soil aggregates to study microbial evolution has been discussed [28]. These aggregates can be formed in the lab and starter cultures can be encapsulated. By arranging microaggregates at different positions, neighborhood composition can be manipulated. A recent advance to control the spatial position of bacteria is the embedding in biocompatible three-dimensional (3D) printing ink and the subsequent printing of "living materials." Using such an approach, researchers demonstrated that an antibiotic-resistant species cross-protected a sensitive species as a consequence of their 3D spatial arrangement [29]. Another interesting opportunity may be offered by the advances in cell engineering to show a programmed aggregation behavior. Thereby, the spatial position of cells can be directly controlled and even manipulated. To measure AE, we need to follow changes in the focal species abundance and activity at a high spatial and temporal resolution. A comparison between well-mixed and structured communities will indicate the role of the spatial arrangement of focal, effector, and modulator species for the emergence of AE.

\section{Stronger consideration of AE in modeling microbial communities}

Also modeling in ecology often assumes species communities with pairwise interactions and HOI only recently received some attention in computational microbial ecology [30]. Interestingly, HOI can emerge in mechanistic models from the underlying biology without the addition of special higherorder terms [4]. A common approach to test the occurrence of HOI involves fitting models of population dynamics or biomass accumulation to competition experiments and evaluating whether pairwise interactions can predict the performance of multispecies systems. Thereby, Mayfield and Stouffer [31] showed that accurate prediction of species survival in a low complexity community composed of eight strains requires the consideration of HOI. However, experimental validation of this approach requires a full factorial design, which is an enormous empirical and statistical challenge. The models of Bairey et al. [30] considered the implications of random HOI and showed that they can increase the stability of diverse microbial communities. What is missing in these models is the consideration of the role of spatial organization in microbial communities and its effects on the strength of HOI. The hypotheses generated by modeling should then be validated in empirical studies using tailored experimental systems mentioned in the previous section.

\section{Implications of AE in microbial ecology}

Bacterial multispecies assemblies carry out crucial functions in engineered and natural systems. However, they still remain black box systems when it comes to the principles that control their assembly and structural and functional stability. This knowledge gap severely limits our capacity to engineer and manage microbial consortia to enable, restore, or improve desired functions.

Although synthetic microbial ecology allows for designing communities with defined properties, they almost exclusively have been arranged in well-mixed culture systems. However, spatial organization has been shown to change the interactions and behavior of bacterial species, and to affect their stress resistance [32]. Establishing the concept of $\mathrm{AE}$ in microbial ecology is an important step to advance our knowledge of fundamental aspects of microbial interactions in complex and structured communities, and to develop consortia that exhibit a long-term stability in dynamic environments where species are exposed to fluctuating conditions, competitors, or predators. Comparable to the application of the concept in agricultural management, investigations of $\mathrm{AE}$ in microbial ecology have the potential to yield ecologically guided concepts for the design of synthetic microbial consortia and guidance for purposeful structuring of their habitats. Designing spatially organized collaborator strains bears the potential to promote and stabilize functions in biotechnological applications or bioremediation approaches.

\section{Conclusion}

The structural and functional differences between microorganisms and "large" organisms often impede the direct applicability of existing ecological theory to microbial ecology [33]. However, theory is necessary to unify observations made in different contexts and to develop a conceptual understanding of the interactions of microorganisms with their biotic and abiotic environment [33]. Microbial communities show distinct spatial patterns, which emerge as a consequence of trophic and energetic interdependencies, and environmental preferences. In the last years, microbial ecology experienced a transition from observational studies on "Who's there?" to more farreaching questions on "Where are they located and why?" and more recently to "What are the consequences?". Following up on this, we propose to adopt the AE concept developed in plant ecology also for microbial communities. This concept should serve as a framework to bundle observations and mechanisms to explain changes in community behavior and stability of diverse communities with complex interactions living in spatially structured assemblages. We provided evidence for the occurrence of $\mathrm{AE}$ in 
microbial ecology and summarized demands for studying AE in microbial communities. This study should serve as an impetus for strengthening future research in spatial microbial ecology by integrating knowledge from related disciplines.

Acknowledgements We thank Canan Karakoç and the reviewers for valuable comments. This work was funded by the Helmholtz Association via the integrated project Controlling Chemicals Fate of the research topic Chemicals in the Environment within the research program Terrestrial Environment.

\section{Compliance with ethical standards}

Competing interests The authors declare no competing interests.

Publisher's note: Springer Nature remains neutral with regard to jurisdictional claims in published maps and institutional affiliations.

\section{References}

1. Lowery NV, Ursell T. Structured environments fundamentally alter dynamics and stability of ecological communities. Proc Natl Acad Sci USA. 2019;116:379-88.

2. Boedicker JQ, Brenner K, Weibel DB. Spatial structure of microbes in nature and the biophysics of cell-cell communication. In: The physical basis of bacterial quorum communication. Springer, 2015; pp 53-81.

3. Watrous JD, Dorrestein PC. Imaging mass spectrometry in microbiology. Nat Rev Microbiol. 2011;9:683.

4. Levine JM, Bascompte J, Adler PB, Allesina S. Beyond pairwise mechanisms of species coexistence in complex communities. Nature. 2017;546:56.

5. Barbosa P, Hines J, Kaplan I, Martinson H, Szczepaniec A, Szendrei Z. Associational resistance and associational susceptibility: having right or wrong neighbors. Annu Rev Ecol Syst. 2009;40:1-20.

6. Underwood N, Inouye BD, Hambäck PA. A conceptual framework for associational effects: when do neighbors matter and how would we know? Q Rev Biol. 2014;89:1-19.

7. Billick I, Case TJ. Higher order interactions in ecological communities: what are they and how can they be detected? Ecology. 1994;75:1529-43.

8. Tecon R, Mitri S, Ciccarese D, Or D, van der Meer JR, Johnson DR. Bridging the holistic-reductionist divide in microbial ecology. mSystems. 2019;4:e00265-00218.

9. Shade A. Diversity is the question, not the answer. ISME J. 2016;11:1.

10. Feng S, Tan CH, Constancias F, Kohli GS, Cohen Y, Rice SA. Predation by Bdellovibrio bacteriovorus significantly reduces viability and alters the microbial community composition of activated sludge flocs and granules. FEMS Microbiol Ecol. 2017;93:fix020.

11. Matz C, Kjelleberg S. Off the hook-how bacteria survive protozoan grazing. Trends Microbiol. 2005;13:302-7.

12. Vidakovic L, Singh PK, Hartmann R, Nadell CD, Drescher K. Dynamic biofilm architecture confers individual and collective mechanisms of viral protection. Nat Microbiol. 2018;3:26.
13. Bossier P, Verstraete W. Triggers for microbial aggregation in activated sludge? Appl Microbiol Biotechnol. 1996;45:1-6.

14. Schmidt R, Cordovez V, de Boer W, Raaijmakers J, Garbeva P. Volatile affairs in microbial interactions. ISME J. 2015;9:2329.

15. Payne P, Geyrhofer L, Barton NH, Bollback JP. CRISPR-based herd immunity can limit phage epidemics in bacterial populations. eLife. 2018;7:e32035.

16. Schulz-Bohm K, Zweers H, de Boer W, Garbeva P. A fragrant neighborhood: volatile mediated bacterial interactions in soil. Front Microbiol. 2015;6:1212.

17. Pande S, Kaftan F, Lang S, Svatoš A, Germerodt S, Kost C. Privatization of cooperative benefits stabilizes mutualistic crossfeeding interactions in spatially structured environments. ISME J. 2015;10:1413.

18. Frost I, Smith WPJ, Mitri S, Millan AS, Davit Y, Osborne JM, et al. Cooperation, competition and antibiotic resistance in bacterial colonies. ISME J. 2018;12:1582-93.

19. Estrela S, Brown SP. Community interactions and spatial structure shape selection on antibiotic resistant lineages. PLoS Comput Biol. 2018;14:e1006179.

20. Luppens S, Kara D, Bandounas L, Jonker M, Wittink F, Bruning $\mathrm{O}$, et al. Effect of Veillonella parvula on the antimicrobial resistance and gene expression of Streptococcus mutans grown in a dual-species biofilm. Oral Microbiol Immun. 2008;23:183-9.

21. Leisner JJ, Jørgensen NO, Middelboe M. Predation and selection for antibiotic resistance in natural environments. Evol Appl. 2016;9:427-34.

22. Bauer WD, Robinson JB. Disruption of bacterial quorum sensing by other organisms. Curr Opin Biotechnol. 2002;13:234-7.

23. Borer B, Tecon R, Or D. Spatial organization of bacterial populations in response to oxygen and carbon counter-gradients in pore networks. Nat Commun. 2018;9:769.

24. Marchal M, Goldschmidt F, Derksen-Müller SN, Panke S, Ackermann M, Johnson DR. A passive mutualistic interaction promotes the evolution of spatial structure within microbial populations. BMC Evol Biol. 2017;17:106.

25. Tecon R, Ebrahimi A, Kleyer H, Erev Levi S, Or D. Cell-to-cell bacterial interactions promoted by drier conditions on soil surfaces. Proc Natl Acad Sci USA. 2018;115:9791-6.

26. Dal Co A, van Vliet S, Kiviet DJ, Schlegel S, Ackermann M. Short-range interactions govern cellular dynamics in microbial multi-genotype systems. bioRxiv. 2019;530584.

27. Aleklett K, Kiers ET, Ohlsson P, Shimizu TS, Caldas VE, Hammer EC. Build your own soil: exploring microfluidics to create microbial habitat structures. ISME J. 2017;12:312.

28. Rillig MC, Muller LA, Lehmann A. Soil aggregates as massively concurrent evolutionary incubators. ISME J. 2017;11:1943.

29. Connell JL, Ritschdorff ET, Whiteley M, Shear JB. 3D printing of microscopic bacterial communities. Proc Natl Acad Sci USA. 2013;110:18380-5.

30. Bairey E, Kelsic ED, Kishony R. High-order species interactions shape ecosystem diversity. Nat Commun. 2016;7:12285.

31. Mayfield MM, Stouffer DB. Higher-order interactions capture unexplained complexity in diverse communities. Nat Ecol Evol. 2017;1:0062

32. Johns NI, Blazejewski T, Gomes ALC, Wang HH. Principles for designing synthetic microbial communities. Curr Opin Microbiol. 2016;31:146-53.

33. Prosser JI, Bohannan BJM, Curtis TP, Ellis RJ, Firestone MK, Freckleton RP, et al. The role of ecological theory in microbial ecology. Nat Rev Microbiol. 2007;5:384. 